\title{
Attitudes, knowledge and consequences of uncertain genetic findings in hypertrophic cardiomyopathy
}

\author{
Charlotte Burns $^{1,2,3}$, Laura Yeates ${ }^{1,3}$, Catherine Spinks ${ }^{1,3}$, Christopher Semsarian ${ }^{1,2,3}$ and Jodie Ingles ${ }^{\star, 1,2,3}$
}

With the surge of next-generation sequencing (NGS) technologies making almost all genetic tests more affordable and available, cardiac genetic testing now routinely encompasses a large number of genes within a panel setting. The additional sensitivity of this practice is limited and has the potential to inflict a spectrum of uncertainty. We sought to explore attitudes, preferences, recall and psychological consequences of informative and uninformative genetic results amongst probands diagnosed with hypertrophic cardiomyopathy (HCM). We conducted semi-structured interviews and analysed the qualitative data using a framework analysis process. In general, we found probands were more concerned with their clinical diagnosis than gene result and in some, recall and understanding of genetic diagnosis was poor. Several participants expected genetic testing would alleviate uncertainty, often holding an altruistic view of participation in testing, removing their sense of self and failing to appreciate fully the familial implications. With the key utility of HCM genetic testing and counselling being for greater risk prediction for at-risk relatives, effective communication within the family is critical. While communication appeared adequate, further questioning found it was often vague, failing to translate into meaningful action by relatives. Based on these findings, a framework of key outcomes to assist multidisciplinary teams in genetic counselling of probands receiving an HCM gene result was developed.

European Journal of Human Genetics (2017) 25, 809-815; doi:10.1038/ejhg.2017.66; published online 3 May 2017

\section{INTRODUCTION}

Hypertrophic cardiomyopathy (HCM) is a heterogeneous disease characterised by unexplained left ventricular hypertrophy in the absence of loading conditions such as hypertension. ${ }^{1}$ With a prevalence of at least 1 in 500 , it is one of the most common inherited heart diseases. ${ }^{2} \mathrm{HCM}$ often presents during adolescence and clinical manifestations range from asymptomatic to impaired diastolic function, heart failure and sudden cardiac death.

HCM is an autosomal dominant disease, with thousands of variants reported as causative in $\sim 15$ sarcomere and sarcomere-related genes. $^{3-5}$ With the surge of next-generation sequencing (NGS) technologies making almost all genetic tests more affordable and available, HCM genetic testing routinely encompasses a comprehensive gene panel. The additional sensitivity of this practice is limited and screening a large number of genes with little or no evidence of HCM association has resulted in a marked increase in detection of variants of uncertain significance (VUS). ${ }^{5}$ The yield of identifying pathogenic/ likely pathogenic variants in HCM is $\sim 30 \%$, ${ }^{6,7}$ while VUS are identified in $15 \%$ but this can be variable depending on the number of genes tested. This VUS rate is not insignificant and has the potential to inflict a spectrum of uncertainty on patients and their families. There is also the risk of identification of incidental genetic findings in other cardiac genes. Incidental or secondary genetic findings occur when compelling variants are identified that are not related to the specific phenotype in question, and is a direct consequence of including more genes in cardiac panels. ${ }^{8}$ The consequences of such a finding can be increased uncertainty, and should be considered during pre-test genetic counselling. In spite of this, limited data exists regarding the patient experience of being faced with such uncertainty. On a background of increased psychosocial difficulties relating to the clinical diagnosis, such as poor health-related quality of life, increased anxiety, and even presence of posttraumatic stress symptoms in some sub-groups, ${ }^{9-12}$ understanding how this uncertainty is dealt with is key.

Few studies focus on the patient perspective regarding uncertain variants, and those that do are often based in the paediatric and cancer genetic settings. While in general most participants report a positive attitude towards receiving results from genetic testing, ${ }^{13-16}$ one recent study examining the attitudes of nearly 7000 health professionals, genomic researchers and the public, highlighted a disconnect between the view of those handling results from NGS testing versus those being tested. ${ }^{14}$ Given the unique challenges HCM patients must face and the increasing yield of uncertain genetic results, we sought to explore the attitudes, preferences, recall and psychosocial consequences of uncertain genetic results.

\section{METHODS}

Participants

HCM probands attending a multidisciplinary specialised HCM clinic in Sydney, Australia who underwent genetic testing and had either (1) a pathogenic or likely pathogenic HCM variant, (2) a VUS or (3) no variant identified, were eligible for inclusion in this study (Figure 1). Pathogenic and likely pathogenic variants were defined as variants with a high probability of affecting function and therefore of causing disease. In families where a pathogenic and likely pathogenic variant was identified, cascade genetic testing of asymptomatic relatives was offered. A VUS was defined as a variant without sufficient evidence to determine pathogenicity and was not used for cascade genetic testing.

\footnotetext{
${ }^{1}$ Agnes Ginges Centre for Molecular Cardiology, Centenary Institute, Sydney, Australia; ${ }^{2}$ Sydney Medical School, University of Sydney, Sydney, Australia; ${ }^{3}$ Department of Cardiology, Royal Prince Alfred Hospital, Sydney, Australia

*Correspondence: Dr J Ingles, Centenary Institute, Agnes Ginges Centre for Molecular Cardiology, Locked Bag 6, Newtown, NSW 2042 , Australia. Tel: +61 2 9565 6293; Fax: +61 29565 6101; E-mail: j.ingles@centenary.org.au

Received 12 December 2016; revised 23 February 2017; accepted 28 March 2017; published online 3 May 2017
} 


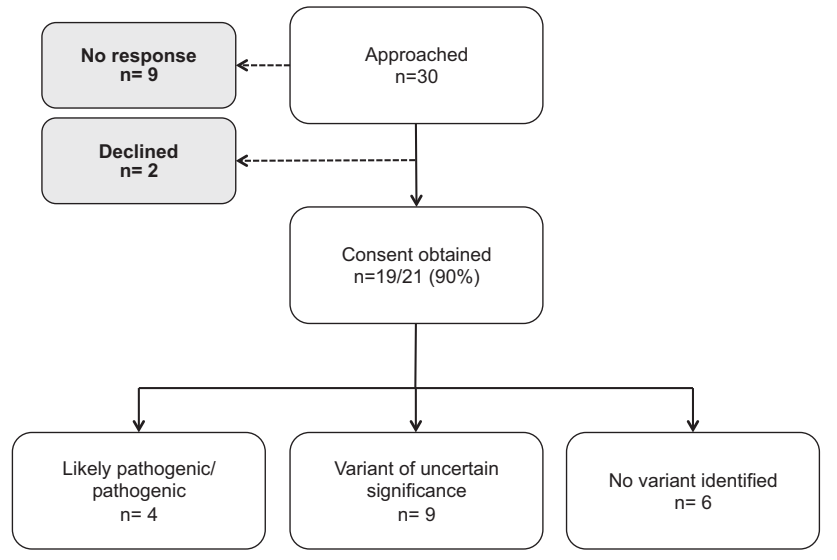

Figure 1 Flowchart of participants approached and recruited to this study.

Eligibility criteria included sufficient English skills, aged 18 years and above and a genetic result disclosed since the 1st January 2014. A purposive sampling method was employed, whereby eligible participants were invited based on a range of demographics and characteristics. A focus was made on recruiting those individuals with uninformative gene results (VUS or no variant identified).

\section{Genetic counselling and testing}

Participants underwent genetic testing and counselling in different ways. The majority (14 participants) had research or commercial-based testing through our multidisciplinary clinic incorporating the expertise of cardiologists and cardiac genetic counsellors, as previously described. ${ }^{17,18}$ The remainder (5 participants) had genetic testing ordered by a Clinical Genetics department, and seen by clinical geneticists and general genetic counsellors in an outpatient hospital setting.

The genetic testing process within the multidisciplinary specialised clinic was as follows (Figure 2). All participants underwent pre and post-test genetic counselling. Pre-test genetic counselling included documentation of the family history, discussion of the process of genetic testing, the potential outcomes and results of testing, and the implications of results for the proband and their family. Results disclosure and post-test counselling was usually within the clinic setting, but occasionally over the phone, and each participant received a brief letter outlining their result. The decision to use research or commercial-based genetic testing was contingent on the residential address of the patient (some health districts will pay for testing) otherwise samples were tested in a research setting. For participants who underwent research testing the pre and post-test genetic counselling process was no different to those undergoing commercial genetic testing, with the exception it may take longer for a gene report to be generated and the additional knowledge they are contributing to improving our overall understanding of disease. The mean time since the result was disclosed for research participants was 1.3 years compared with 1.8 years for those who underwent clinical genetic testing. Research-based testing included clinical interpretation of variants in genes with unequivocal evidence of disease association only. Participants were consented that any information of clinical relevance to them will be returned and pre and post-test genetic counselling performed by experienced cardiac genetic counsellors. ${ }^{18}$

\section{Variant classification and nomenclature}

Participants variants were classified using in-house criteria based on the updated American College of Medical Genetics (ACMG) guidelines (see ClinVar, Agnes Ginges Centre for Molecular Cardiology variant assessment and assertion criteria; https://submit.ncbi.nlm.nih.gov/ft/byid/djgybgii/mdi5363_505375_agnesginges_variantassess_clinvar.pdf). ${ }^{19}$ Key determinants of pathogenicity included rarity (minor allele frequency of $\leq 0.02 \%$ ) or absence from the ExAC data set, previous reports of the variant in 2 or more additional unrelated HCM patients (http://ncbi.nlm.nih.gov/pubmed and http://clinvar. $\mathrm{com} /$ ), segregation with affected relatives where possible, as well as any

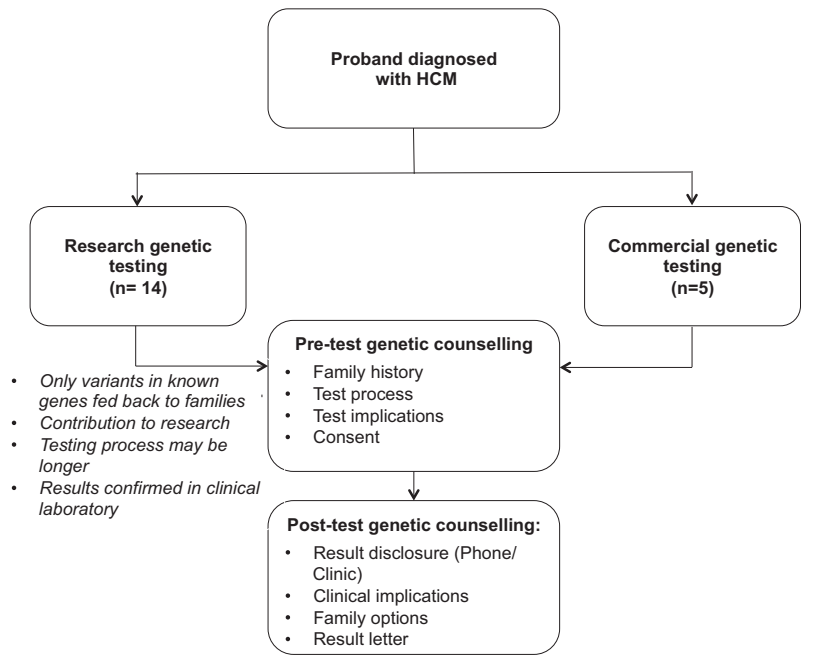

Figure 2 The process of cardiac genetic counselling.

supportive experimental data. Overall agreement amongst in silico tools and conservation scores was considered a single low-level supportive criterion. Variants have been prefaced with the appropriate classification adjective throughout the manuscript. Classifications included pathogenic (class V equivalent), likely pathogenic (class IV equivalent) and variant of uncertain significance (Class III equivalent).

\section{Study design}

A qualitative interview study design was used to explore a number of factors across the different result scenarios. The interview scedule included experiences of dealing with genetic uncertainty, knowledge and recall of complex genetic information and attitudes, preferences and psychosocial consequences of an uncertain genetic result. Semi-structured interviews were conducted face-to-face $(n=1)$ or by phone $(n=18)$, as preferred by the participant. An interview schedule informed by the relevant literature and reviewed by the multidisciplinary team provided consistency between interviews and was reviewed and amended to include emerging themes in the first stage of thematic analysis. A cardiac genetic counsellor (CB) conducted the interviews between May 2016 and August 2016, and had not been involved in any pre or post-test genetic counselling of participants. The preliminary findings and framework development were discussed amongst four cardiac genetic counsellors throughout the data collection process (CB, CSp, LY and JI). Interviews continued until thematic saturation was reached.

\section{Analysis}

Audio-recordings were transcribed verbatim, de-identified and coded using a frame-work analysis process. Each transcript was reviewed by the primary researcher (CB). Initial themes were discussed amongst the author group. The data were coded according to the agreed framework. Each transcript was reviewed by CB with $11(58 \%)$ double coded by LY. A further three transcripts (16\%) were reviewed by an additional cardiac genetic counsellor (CSp). We used an iterative process to refine the framework during data collection and analysis. The data were summarised with supporting quotes in an excel spreadsheet.

\section{RESULTS}

\section{Participants}

There were 19 HCM probands who completed interviews (Table 1). Eleven $(58 \%)$ were male, with a mean age of $54 \pm 13$ years and $8(42 \%)$ had university level education or higher. Fifteen participants $(79 \%)$ had gene results considered to be uninformative, including $9(47 \%)$ VUS and $6(32 \%)$ where no variant was identified. We purposively enriched our patient sample for those 
Table 1 Participant characteristics

\begin{tabular}{|c|c|}
\hline Characteristic & Sample (\%) \\
\hline$n$ & 19 \\
\hline Male gender, $n(\%)$ & $11(58)$ \\
\hline Mean age, years $\pm S D$ & $54 \pm 13$ \\
\hline \multicolumn{2}{|l|}{ Result classification, $n$ (\%) } \\
\hline Pathogenic/Likely pathogenic & $4(21)$ \\
\hline Variant of uncertain significance & $9(47)$ \\
\hline No variant identified & $6(32)$ \\
\hline Research result, $n(\%)$ & $14(74)$ \\
\hline Median time since blood collected, years (range) & $3(1-14)$ \\
\hline Research & $5(2-14)$ \\
\hline Clinical & $2(1-5)$ \\
\hline Time since result returned, years (range) & $1(1-2)$ \\
\hline Research & $1(1-2)$ \\
\hline Clinical & $2(1-2)$ \\
\hline Family history of $\mathrm{HCM}, n(\%)$ & $9(47)$ \\
\hline \multicolumn{2}{|l|}{ Education level, $n(\%)$} \\
\hline Postgraduate/University & $8(42)$ \\
\hline High School/Diploma & $8(42)$ \\
\hline Year 10 or below & $3(16)$ \\
\hline Mean interview duration, minutes \pm SD & $20 \pm 11$ \\
\hline
\end{tabular}

with uninformative results and continued recruitment until we were satisfied thematic saturation was complete. The majority of participants completed genetic testing as part of a research programme $(74 \%)$. The mean age at diagnosis of participants was $42 \pm 16$ years. The mean maximal left ventricular wall thickness was $19 \pm 5 \mathrm{~mm}$. Nine $(47 \%)$ probands had a family history of HCM and one had a personal history of resuscitated cardiac arrest.

\section{Overview of results}

There were three key themes that emerged during the interviews. These will be further explored under the subheadings:

(1) Individual experiences with HCM genetic testing and results.

(2) Knowledge and recall of complex genetic information.

(3) Communication and the value of information.

Further, those with uninformative results reported unique issues that are explored separately under a 4 th heading.

More generally, the impact of HCM genetic testing was highly dependant on the context, time and place that testing and disclosure of results took place. This was particularly apparent amongst probands with an uninformative result performed in a research setting, where genetic testing blended into 'everything else' regarding their diagnosis. Of those participants approached for interview, four (two of whom went on to be interviewed) with an uninformative result had no recollection of having genetic testing, in spite of pre and post-test genetic counselling. Further, it appeared that although most patients had adequate conceptualisation of results, translation into meaningful action (such as screening or genetic counselling) by relatives was minimal. Recall and understanding of results was poorest amongst those probands who received uninformative results.

Individual experiences with genetic testing and results. Context, time and place: The impact of genetic testing was highly dependent on the context time and place. Often patients referred to the 'traumatic shock' of diagnosis and, genetic testing, which followed many years later had a comparatively smaller impact. Participants often recounted the story of their diagnosis when asked about their experience with genetic testing.

I'm probably saying things now in a calmer, more reasoned way than I did at the time. I can remember, you know, telling family when I first got picked up, prior to genetic testing and being quite, I suppose traumatised, certainly

P4, Male (51 years), VUS

I think because I was diagnosed with it quite a few years ago, so anything around hypertrophic cardiomyopathy I've accepted

P9, Female (31 years), no variant identified

Perceived value of genetic testing: Participants' perceptions regarding the value of genetic testing varied, many of those with uninformative results viewed genetics as an altruistic contribution to research rather than a test, which may have personal and familial implications. This highlighted a need for genetics professionals to even further emphasise the familial aspect of testing when participants are undergoing research-based testing. Given the large proportion of participants having their genetic testing performed as part of research, it is unclear whether this is a theme unique to research participants or to anyone in general undergoing HCM genetic testing.

I guess the real reason comes right down to it is, I know that, you know, genetics studies we always need, we always need volunteers and we always need more data and the more the data the better

P8, Male (56 years), VUS

And it was the suggestion that this would be a great opportunity to help ... and I thought, you know... it wasn't a big deal

P14, Male (55 years), Likely Pathogenic

Those with informative results however, were more likely to consider children and future generations when reflecting on the value of genetic testing. This group were able to identify the more tangible benefits to future generations, compared with those with uninformative results. One participant with an uninformative result seemed only to consider the impact to children important had the results been informative.

I suppose that for my kids it would be now, um if they chose to have children and get them genetic tested

P1, Female (47 years), Pathogenic

Yeah, so the finding out, finding out was just, "Oh, ok", confirmation and that just, um, you know, doubly reassures the need for my children to have to be checked out

P14, Male (55 years), Likely Pathogenic

I was a bit hesitant because, obviously the implications for my children if I was found to have one that was actually mapped that they could identify it was a gene issue

P11, Female (60 years) no variant identified

Sense of self removed from genetic results: Familial or personal implications of genetic test results were often not considered by participants. This appeared to be the case, more so, for those with 
uninformative results, although also for some with informative results. At time of blood collection, patients undergo pre-test genetic counselling to explain the process of genetic testing, the potential outcomes and impact for the family. If they decide to go ahead with testing, whether research or commercial, they will sign a consent form.

Genetics side of it, um, there wasn't a lot to remember because I only had to give blood

P10, Male (63 years), VUS

Oh well I thought it was, I just think it's a great thing you guys are doing so if I could help I'm all for it, if I'm asked to provide any more blood tests or anything else I'm happy to oblige

P16, Male (59 years), VUS

Because I was asked would I be interested and these are some of the reasons why, um for you to consider... which was helping research and that sort of thing, I thought I would help out

P14, Male (55 years), Likely Pathogenic

Perceived certainty in their diagnosis and finding an answer: The idea that genetic testing would provide an answer and clarity regarding an individual's diagnosis was raised by a number of participants. No participant reflected on the potential for genetic testing to remain unresolved. A positive gene result was seen as a confirmation of their clinical diagnosis and an explanation for familial inheritance.

I was pretty pleased really, that there was a gene that had been, isolated and found to explain my cardiac condition and also I was pleased because it meant both my mum and I then had, you know, an answer for why, for why we get these, um, palpitations.

P3, Female (26 years), Likely Pathogenic

Suggested ah, that genetic testing, well, that the blood test would, $100 \%$ affirm what happened... do I have the blood test and, ah, and what's the cost and the insurance issues was the other issue.... So there was this series of decisions and thoughts and things like that

P14, Male (55 years), Likely Pathogenic

Knowledge and recall of complex genetic information. Overall, the knowledge and recall of the result was dependent on the result classification, person, and the context. Amongst the group there was generally sufficient conceptualisation, particularly for those where a causative variant was identified.

Ah, so I guess, so, the main thing I would say is that the gene that was isolated which, which I can't actually remember the exact transcription of it, it's PK, ah, hold on, I don't even have it... Well I guess the main thing is that I recall from it was that it was an autosomal dominant pathway sort of, and so that with my children- and the main thing that really stuck in my mind was that my children would have a 50:50 chance of getting the gene

P3, Female (26 years), Likely Pathogenic

However discrepancies were apparent at an individual level, with limited understanding of the implications for family members by some participants. It appeared those with uninformative results were less clear in their conceptualisation and recall of genetic results. Further, the idea that their uninformative results was able to rule out possible diagnoses, highlighted poor understanding and confusion.
At least hopefully you can rule some things out P15, Female (52 years), no variant identified

I don't exactly understand what you mean by genetics P19, Male (57 years), VUS

I think it goes a long way to sort of settling in your mind what I've got

P7, Female (72 years), VUS

Lack of confidence in genetics understanding: Throughout many of the interviews participants sought validation of their knowledge, asking questions during and after the interview. This highlighted the importance of information and resources and ensuring probands feel adequately equipped and supported to pass the appropriate knowledge on to family members.

Would it be an idea to send my latest genetic result to her so she can have it in case she needs it, you know, take it to her doctor?

P7, Female (72 years), VUS

So, and again, this is my understanding versus being open to you correcting me if I've not got it right

P4, Male (52 years), VUS

So from what I've sort of answered in the interview here do you consider that I've got a reasonably good understanding of, of what I've been through in terms of testing?

P4, Male (52 years), VUS

Communication. When asked about informing relatives of the genetic risk or result in the family, communication appeared to be generally good though this did not always translate to meaningful action by relatives such as clinical screening or referral to genetics services where appropriate. It appeared effective communication might be problematic for some, and the explanation by the proband about what family members had been told was often vague. For those with a causative variant identified, this did seem to be less of an issue.

Yeah I don't think I've actually, I haven't mentioned it to anybody P15, Female (52 years), no variant identified

Many years ago I did contact them to say, blah, blah, blah. Yeah, so they're... I mean, it's not a secret

P16, Male (59 years), VUS

I was thinking, um, maybe I'd sort of get my sisters to start going down once every 2 years

P13, Male (37 years), no variant identified

Ah, no, I have a very supportive family and, and actually my, I've had my father and my mother and my husband, all three of the key people in my life come to cardiac appointments with me

P3, Female (26 years), Likely Pathogenic

The value of information: Throughout many of the interviews, the importance of periodic recontact with the team alongside resources to assist with understanding and communication was raised. 


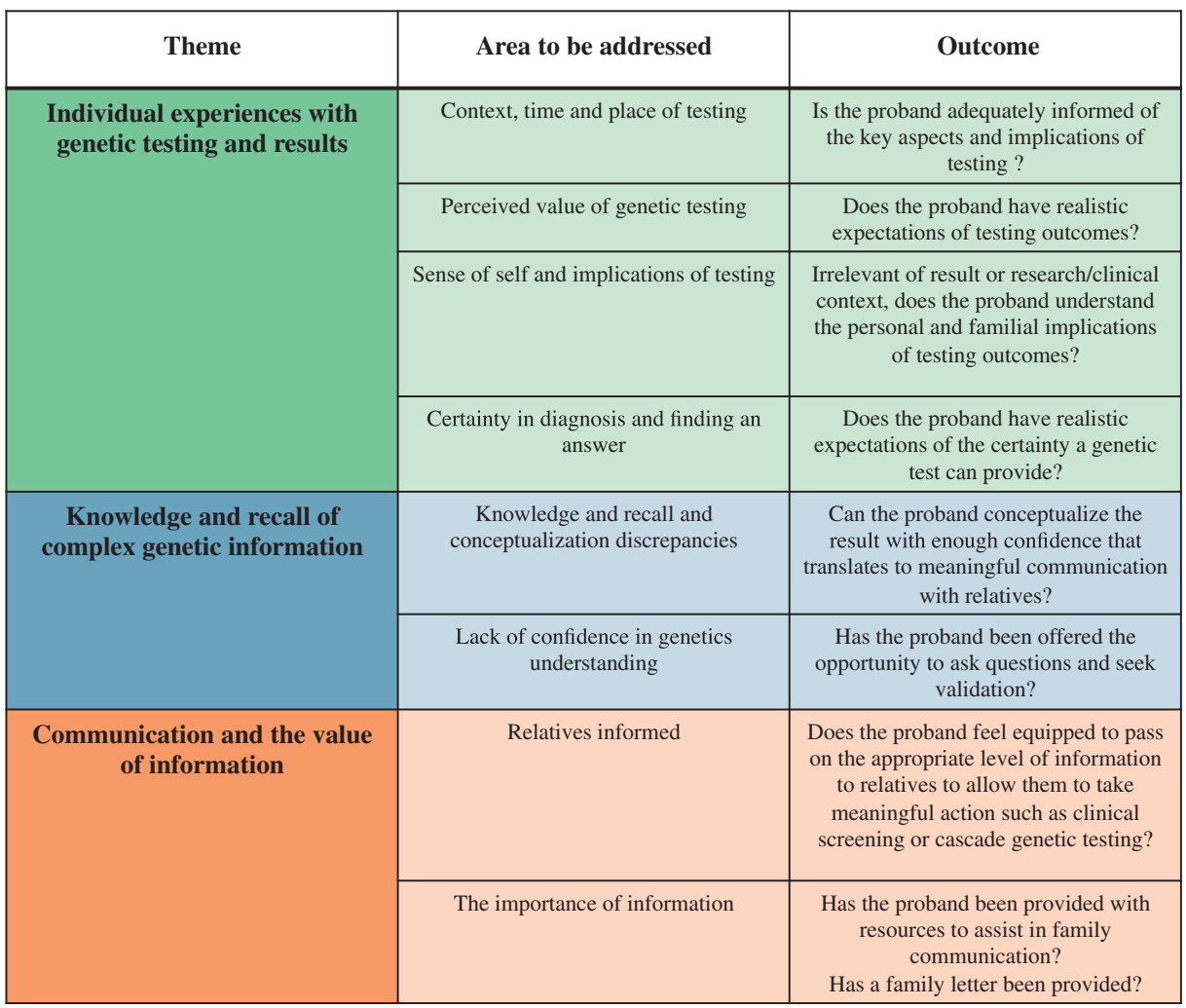

Figure 3 Framework for addressing key areas within genetic counselling communication with the proband.

So that would probably be my only comment on that and maybe, you know, I probably would've liked a little bit more detail perhaps if that was possible

P11, Female (60 years) no variant identified

It was very helpful because, absolutely, because, as you heard, I'm not good at remembering the gene, so I have had to refer to my letter on a few occasions

P3, Female (26 years), Likely Pathogenic

Something saying here's the facts, here's the implications, here's some suggestions and recommendations

P14, Male (55 years), Likely Pathogenic

Variants of uncertain significance group. Those with an uncertain variant may require additional information when receiving results and pre-test counselling should carefully consider the potential of unveiling uncertain genetic variants. This group often perceived they might have a different type of HCM, and their recall and conceptualisation of results appeared poorer.

Right, and it's a bit that way I guess with, with myself, we don't know what has caused the HOCM, to a certain degree I suspect it hasn't, just because mine is so minor, do you get what I mean?

P8, Male (55 years), VUS

Yes it did because it was just so unusual for want of a better word right now, I can't think of one, it was horrific in a way because, '[Oh my god, my heart is really bad now]

P7, Female (72 years), VUS
Look I think I'm a little bit still on the fence because of the, the fact that I, although I fit into the characteristics as I understand it, there's still not a clear link and if I've got this correct, and please, please offer the contrary if I'm not, but some, some people that have been picked up with HCM because it's such a wide variety, have a more definite, have a more definite understanding of it because of the testing that has been done in their situation

P4, Male (52 years), VUS

Framework for improving communication to the proband

Based on the themes collated from our data, by targeting key areas in communication with the proband, and in paying particular attention to those with uncertain results in pre- and post-test counselling, improved action by relatives may be achieved. In targeting these areas, genetic counsellors and the multidisciplinary team could maximize effective communication of genetic risk and results to probands. This may improve the translation of these conversations into meaningful action by relatives. Key outcomes within these areas have been summarised based on discussions with the multidisciplinary team after review of transcripts. A framework to guide development of a resource that aims to ensure sufficient conceptualisation of genetic test results and empower the proband to communicate risk to relatives has been developed (Figure 3).

\section{DISCUSSION}

Overall participants were more concerned about their clinical diagnosis than information arising from genetic testing. In many cases, particularly those who had research-based genetic testing, recall and understanding of genetic information was poor. A number of participants expected genetic testing would provide clarity and were 
unprepared for additional uncertainty. Many often held an altruistic view of their participation in genetic research, removing their sense of self and failing to fully appreciate the familial implications of their results. In others, particularly those with an uncertain genetic result, genetic recall was poor and either affirmed a belief they had something 'different' to HCM, or was incorrectly perceived as reassuring for other relatives. Whilst these issues were not problematic for every participant, the key utility of genetic testing in HCM is for identification of at-risk relatives. Therefore, clear communication between the proband and at-risk relatives is a critical step in this pathway and one not being adequately addressed at present.

Theories of health behaviour and stress are relevant to genetic testing research. The transactional model of stress and coping and the theory of planned behaviour suggest an individuals ability to cope and the impact of genetic results or decision to undergo testing may be influenced by personal characteristics and coping style. Specifically these may be influenced by their need for certainty, information seeking preferences and sense of control over health outcomes. ${ }^{20,21}$ The need for certainty is often cited as the primary benefit in the uptake of testing across a number of genetic testing contexts, an interesting consideration given the uncertainty inherent to the practice. $^{22-24}$ Certainty and the idea of tangible treatment options are not always achievable and this misperception of the test highlights the need for even more careful pre-test genetic counselling. ${ }^{24}$

The patient perspective regarding uncertain genetic findings in the setting of HCM is currently understudied. To date, the focus has largely been around what laboratories and clinicians would prefer to see on a report, with little attention to how the patient will respond to this information. A recent study exploring the informed consent process amongst individuals with a Mendelian disease undergoing whole exome/genome research testing concluded this group might be more likely to inadequately consider their risk due to their strong desire to find an answer and help advance research. ${ }^{25}$ Given the value of genetic testing for many people is to reduce uncertainty, understanding the impact of uncertain results and ensuring informed consent is guided by appropriate research and theory is vital. ${ }^{26}$ The current practice (which does vary between laboratories) that often includes reporting of uncertain variants is one that should be carefully reconsidered. Uncertain variants that are highly suspicious and where additional segregation studies may help to clarify its role are an exception, however where it cannot be avoided, measures to better prepare patients and ensure correct understanding need to be implemented.

We have highlighted a number of conceptualisation issues relating to the gene result. Many participants value the perceived certainty a genetic test offers, in spite of increasingly uncertain and complex results. Further, some assign incorrect meaning of a result, particularly where it is an uncertain or uninformative result. In addition, the familial implications, although understood at a superficial level are often not translated in to meaningful action by relatives. Having adequate recall of the outcome of genetic testing, whether it be positive, no variant identified or uncertain is a critical first step in ensuring appropriate information is communicated to at-risk relatives. In the general genetic literature, various studies have demonstrated issues with recall and personal interpretation regarding genetic results, leading to families inaccurately understanding their meaning. ${ }^{27,28}$ Indeed, poor understanding has been shown to impact on communication of risk to family members. ${ }^{29}$ The consent process and pre-test expectations are therefore important considerations when examining the patient's ability to cope with uncertain results. ${ }^{15,30}$ The need for additional information or support could provide important targets for interventions to improve pre-test genetic counselling in this setting.

Given the research participants were predominantly from a highly educated background, it could be speculated that they represent a highly literate and engaged patient population. Our findings may therefore be an overestimate of the true level of recall and understanding of HCM genetic test results amongst the HCM population. Health disparities between high and low socioeconomic status patients are well known ${ }^{31,32}$ and genetics is likely an area that could increase these disparities. ${ }^{33}$ Methods to better support patient understanding and decision-making, tailored to the needs of all patients, is therefore likely to be of even greater importance.

To address these issues, it does not appear that a 'one-size fits all' approach applies. The need for a personalised and evidence-based consent and communication process is clear. Where informational needs of patients and their families are not fully met, particularly for families who receive uncertain results, additional genetic counselling or information would be beneficial, ${ }^{34}$ and indeed providing appropriate support, genetic counselling and resources are effective in improving comprehension. ${ }^{27,34,35}$ The lack of confidence the patients demonstrated regarding their genetic knowledge, highlights our current practice is falling short. We have developed a framework to guide more effective pre- and post-test cardiac genetic counselling. Key 'problem' areas identified have been translated into 'areas to be addressed' and followed by the proposed outcome. It is hoped such a framework will guide genetic counsellors, or be used in the development of resources, to allow more effective communication.

In summary, HCM patients undergoing proband genetic counselling may require additional support and information above current practice. The key role of HCM genetic testing is for the at-risk family members, and therefore effective communication with the family is vital. Further, inclusion of uncertain variants on gene reports should be carefully considered. Efforts to ensure the proband is sufficiently empowered to communicate effectively with family members should be considered and we have developed a framework aimed at addressing these key areas.

\section{CONFLICT OF INTEREST}

The authors declare no conflict of interest.

\section{ACKNOWLEDGEMENTS}

CB is the recipient of an Australian Postgraduate Award (APA). CS is the recipient of a National Health and Medical Research Council (NHMRC) Practitioner Fellowship (\#1059156). JI is the recipient of a National Heart Foundation of Australia Future Leader Fellowship (\#100833). This study is funded in part by an NHMRC Project Grant (\#1059515).

\footnotetext{
1 Maron BJ: Hypertrophic cardiomyopathy: a systematic review. JAMA 2002; 287: 1308-1320.

2 Semsarian C, Ingles J, Maron MS, Maron BJ: New perspectives on the prevalence of hypertrophic cardiomyopathy. J Am Coll Cardiol 2015; 65: 1249-1254.

3 Alfares AA, Kelly MA, McDermott G et al: Results of clinical genetic testing of 2,912 probands with hypertrophic cardiomyopathy: expanded panels offer limited additional sensitivity. Genet Med 2015; 17: 880-888.

4 Ackerman MJ, Priori SG, Willems S et al: HRS/EHRA expert consensus statement on the state of genetic testing for the channelopathies and cardiomyopathies (vol 13, pg 1077, 2011). Europace 2012; 14: 277-277.

5 Maron BJ, Maron MS, Semsarian C: Genetics of hypertrophic cardiomyopathy after 20 years: clinical perspectives. J Am Coll Cardiol 2012; 60: 705-715.

6 Alfares AA, Kelly MA, McDermott $G$ et al: Results of clinical genetic testing of 2,912 probands with hypertrophic cardiomyopathy: expanded panels offer limited additional sensitivity. Genet Med 2015; 17: 880-888.
} 
7 Ingles J, Sarina T, Yeates L et al: Clinical predictors of genetic testing outcomes in hypertrophic cardiomyopathy. Genet Med 2013; 15: 972-977.

8 Ingles J, Burns C, Barratt A, Semsarian C: Application of genetic testing in hypertrophic cardiomyopathy for preclinical disease detection. Circ Cardiovasc Genet 2015; 8: 852-859.

9 Hamang A, Eide GE, Nordin K, Rokne B, Bjorvatn C, Oyen N: Health status in patients at risk of inherited arrhythmias and sudden unexpected death compared to the general population. BMC Med Genet 2010; 11: 27.

10 Ingles J, Yeates L, Hunt L et al: Health status of cardiac genetic disease patients and their at-risk relatives. Int J Cardiol 2013; 165: 448-453.

11 Cox S, O'Donoghue AC, McKenna WJ, Steptoe A: Health related quality of life and psychological wellbeing in patients with hypertrophic cardiomyopathy. Heart 1997; 78 182-187.

12 Ingles J, Sarina T, Kasparian N, Semsarian C: Psychological wellbeing and posttrau matic stress associated with implantable cardioverter defibrillator therapy in young adults with genetic heart disease. Int J Cardiol 2013; 168: 3779-3784.

13 Facio FM, Eidem H, Fisher $\mathrm{T}$ et al: Intentions to receive individual results from wholegenome sequencing among participants in the ClinSeq study. Eur J Hum Genet 2013; 21: 261-265.

14 Middleton A, Morley KI, Bragin E et al: Attitudes of nearly 7000 health professionals, genomic researchers and publics toward the return of incidental results from sequencing research. Eur J Hum Genet 2016; 24: 21-29.

15 Meiser B, Storey B, Quinn V, Rahman B, Andrews L: Acceptability of, and information needs regarding, next-generation sequencing in people tested for hereditary cancer: a qualitative study. J Genet Counsel 2016; 25: 218-227.

16 Fernandez CV, Bouffet E, Malkin D et al: Attitudes of parents toward the return of targeted and incidental genomic research findings in children. Genet Med 2014; 16: 633-640.

17 Ingles J, Lind JM, Phongsavan P, Semsarian C: Psychosocial impact of specialized cardiac genetic clinics for hypertrophic cardiomyopathy. Genet Med 2008; 10 : 117-120.

18 Ingles J, Semsarian C: Conveying a probabilistic genetic test result to families with an inherited heart disease. Heart Rhythm 2014; 11: 1073-1078.

19 Richards S, Aziz N, Bale S et al: Standards and guidelines for the interpretation of sequence variants: a joint consensus recommendation of the American College of Medical Genetics and Genomics and the Association for Molecular Pathology. Genet Med 2015; 17: 405-424.

20 Lazarus RS: Coping theory and research: past, present, and future. Psychosomat Med 1993; 55: 234-247
21 Ajzen I: The theory of planned behavior. Organ Behav Hum Dec 1991; 50: 179-211.

22 Croyle RT, Dutson DS, Tran VT, Sun YC: Need for certainty and interest in genetic testing. Womens Health 1995; 1: 329-339.

23 Gooding HC, Organista K, Burack J, Biesecker BB: Genetic susceptibility testing from a stress and coping perspective. Soc Sci Med 2006; 62: 1880-1890.

24 Press NA, Yasui Y, Reynolds S, Durfy SJ, Burke W: Women's interest in genetic testing for breast cancer susceptibility may be based on unrealistic expectations. Am J Med Genet 2001; 99: 99-110.

25 Bergner AL, Bollinger J, Raraigh KS et al: Informed consent for exome sequencing research in families with genetic disease: the emerging issue of incidental findings. $A m$ J Med Genet A 2014; 164A: 2745-2752.

26 Fanos JH: New 'first families': the psychosocial impact of new genetic technologies. Genet Med 2012; 14: 189-190.

27 Kiedrowski LA, Owens KM, Yashar BM, Schuette JL: Parents' perspectives on variants of uncertain significance from chromosome microarray analysis. J Genet Counsel 2016; 25: 101-111.

28 Turbitt E, Halliday JL, Amor DJ, Metcalfe SA: Preferences for results from genomic microarrays: comparing parents and health care providers. Clin Genet 2015; 87 21-29.

29 Daly MB, Montgomery S, Bingler R, Ruth K: Communicating genetic test results within the family: Is it lost in translation? A survey of relatives in the randomized sixstep study. Fam Cancer 2016; 15: 697-706.

30 O'Neill SC, Rini C, Goldsmith RE, Valdimarsdottir H, Cohen LH, Schwartz MD: Distress among women receiving uninformative BRCA1/2 results: $12-$ month outcomes. Psychooncology 2009; 18: 1088-1096.

31 Korda RJ, Butler JR, Clements MS, Kunitz SJ: Differential impacts of health care in Australia: trend analysis of socioeconomic inequalities in avoidable mortality. Int $J$ Epidemiol 2007; 36: 157-165.

32 van Doorslaer E, Masseria C, Koolman XGroup OHER: Inequalities in access to medical care by income in developed countries. CMAJ 2006; 174: 177-183.

33 Ingles J, Johnson R, Sarina T et al: Social determinants of health in the setting of hypertrophic cardiomyopathy. Int J Cardiol 2015; 184: 743-749.

34 Reiff M, Bernhardt BA, Mulchandani S et al: 'What does it mean?': uncertainties in understanding results of chromosomal microarray testing. Genet Med Genet 2012; 14 250-258.

$35 \mathrm{Jez}$ S, Martin M, South S, Vanzo R, Rothwell E: Variants of unknown significance on chromosomal microarray analysis: parental perspectives. J Community Genet 2015; 6 : 343-349. 